https://doi.org/10.1109/IGARSS39084.2020.9323455

https://archimer.ifremer.fr/doc/00719/83135/

\title{
CAL/VAL Phase for the Swim Instrument Onboard cFOSAT
}

Tourain C. ${ }^{1}$, Hauser D. ${ }^{2}$, Hermozo L. ${ }^{1}$, Suquet R. Rodriguez ${ }^{1}$, Schippers P. ${ }^{2}$, Aouf L. ${ }^{4}$, Dalphinet A. ${ }^{4}$, Mouche Alexis ${ }^{5}$, Chapron Bertrand ${ }^{5}$, Collard Fabrice ${ }^{5}$, Dufour C. ${ }^{2}$, Gouillon F. ${ }^{1}$, Ollivier A. 7 , Piras F. ${ }^{6}$, Dalila M. ${ }^{6}$, Guitton Gilles ${ }^{5}$, Lachiver J-M ${ }^{1}$, Tison C. ${ }^{1}$

${ }^{1}$ CNES, 18 Ave Edouard Belin, F-31400 Toulouse, France.

2 Sorbonne Univ, LATMOS, CNRS, UVSQ, 11 Blvd dAlembert, F-78280 Guyancourt, France.

${ }^{3}$ ACRI ST, 260 Route Pin Montard, F-06904 Biot, France.

${ }^{4}$ Meteo France, 42 Ave Gaspard Coriolis, F-31057 Toulouse, France.

5 IFREMER, ZI Pointe Diable, F-29280 Plouzane, France.

6 Ocean Datalab, F-29280 Plouzane, France.

${ }^{7}$ CLS, Parc Technol Canal, F-31520 Ramonville St Agne, France.

\begin{abstract}
:
The Chinese-French oceanography satellite, CFOSAT, was launched on October 2018. Two Ku-band scatterometers are on-board: SCAT for the wind observation and SWIM for the wave observation. This paper presents the most recent results on the SWIM data quality analysis a few months after the end of the CAL/VAL phase.
\end{abstract}

Keywords : radar, ocean waves, scatterometer, CFOSAT, SWIM 


\section{INTRODUCTION}

The CFOSAT mission was successfully launched on October, 29 $9^{\text {th }}$ 2018. Thanks to a fruitful Chinese-French cooperation, this mission opens a new era in oceanography, allowing the combined measurement and monitoring of wind and waves. CFOSAT offers promising prospects such as improving atmospheric and oceanographic models and predictions, and refining studies of extreme events forecasting by assimilating new data. CFOSAT also brings key observations to better understand ocean/atmosphere interactions, which play a critical role in the climate system.

CFOSAT is composed of two radar sensors both scanning in azimuth: SCAT, a fan-beam wind scatterometer [1], and SWIM designed for wave measurements. This paper focuses on the SWIM measurements only. SWIM is an innovative $\mathrm{Ku}$-band real-aperture wave scatterometer, with 6 low-incidence rotating beams [2]. The performance of the instrument and the quality of the main products based on the CALibration and VALidation (CAL/VAL hereafter) phase are described in details in [3]. Here we summarize these results and report on additional analyses carried out in the prospect of improving the data processor for its upcoming version. SWIM delivers information from its nadir beam (significant wave height, wind speed), and from its 5 off-nadir beams (ocean wave spectra and normalized radar cross- section $\sigma_{0}$ profiles). The main results for each type of product are presented in the following sections. Details on these products can be found in [2], [3].

\section{NADIR BEAM PARAMETERS}

Over ocean surfaces, the main nadir geophysical products are the significant wave height (SWH), the normalized radarcross-section $\sigma_{0}$, and the wind speed (WS). An innovative algorithm, namely 'Adaptive retracking' is implemented in the CFOSAT French ground segment [4]. The retracking is performed at the rate of the nadir echo acquisition (every $220 \mathrm{~ms}$ in the nominal mode of SWIM acquisition), and the geophysical products are provided either as "native" values (at $4.5 \mathrm{~Hz}$ ), or as averaged values (1s, or $4.5 \mathrm{~s}$ per wave box).

The comparison of Significant Wave Height (SWH) between SWIM and collocated model data (ECMWF WAM) shows a very good consistency with a very weak and stable bias: less than $1 \mathrm{~cm}$ with respect to ECMWF over 6 months (Fig.1). This bias is only slightly variable with wave height, the dependency with wind is almost negligible. The comparison with the altimetry mission Jason 3 (Fig. 2-left panel) also highlights a very good consistency, with a mean difference in SWH of less than $1 \mathrm{~cm}$ (and a $35 \mathrm{~cm}$ standard deviation). The SWIM nadir normalized radar cross-section $\sigma_{0}$ also shows a remarkable consistency with the Jason $3 \mathrm{Ku}-$ Band instrument (Fig. 2- right panel) with a mean difference of about $0.12 \mathrm{~dB}$ (standard deviation of $0.4 \mathrm{~dB}$ ). The nadir wind speed provided in the SWIM products It is compliant with the specifications of $2 \mathrm{~m} / \mathrm{s}$ with a mean bias of about $0.10 \mathrm{~m} / \mathrm{s}$ and a rms error of the order of $1 \mathrm{~m} / \mathrm{s}$ with respect to ECMWF wind model data. The results summarized above were obtained with almost no data rejection except those corresponding to a non-converging MLE estimation in the retracking procedure.

This type of analysis repeated on a regular basis revealed that in some occasions (a few percentage of the time), some return samples are missing in the acquisition and in the 
averaging operation applied in real time on the echo power. The source of this problem was identified as being due to intermittent micro-cuts in the electronic system of the rotating part of the antenna system. Without any correction, the main impact of this problem is a short and temporary drop in the $\sigma_{0}$ values, as the real-time averaged value of $\sigma_{0}$ is estimated on an overestimated number of samples. This mis-functioning is now well identified and characterized (Fig. 3). After a first event in June 2019, and an increase which started mid-October 2019, the occurrence is currently almost stable. It occurs 3 to $10 \%$ of the time for all beams except for the nadir beams where the occurrence reaches 15 to $20 \%$ of the time. These statistics were obtained by analyzing drops of the backscatter power integrated over the footprint exceeding $1 \mathrm{~dB}$ between successive macrocycles. For the next version of the ground-processing, these data will be flagged and not included in the $1 \mathrm{~Hz}$ and $5 \mathrm{~Hz}$ mean values, in order to avoid punctual underestimation of $\sigma_{0}$ (and overestimation of wind speed) from the nadir observations. It was verified that this problem has no impact on the SWH values. Note however that the missing samples in the data induces an increase of the standard deviation on $\sigma_{0}$ and SWH.

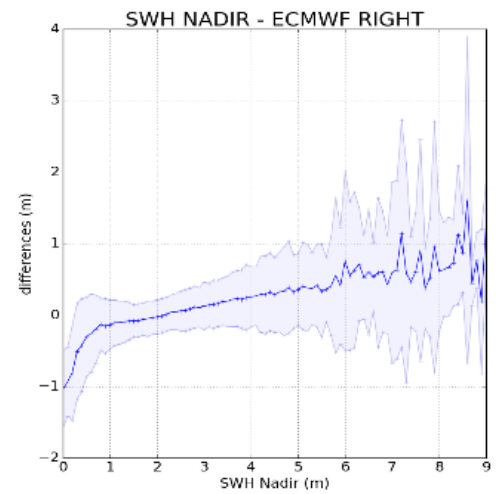

Figure 1: Difference between SWIM nadir SWH and ECMWF SWH trend, as a function of SWH values.
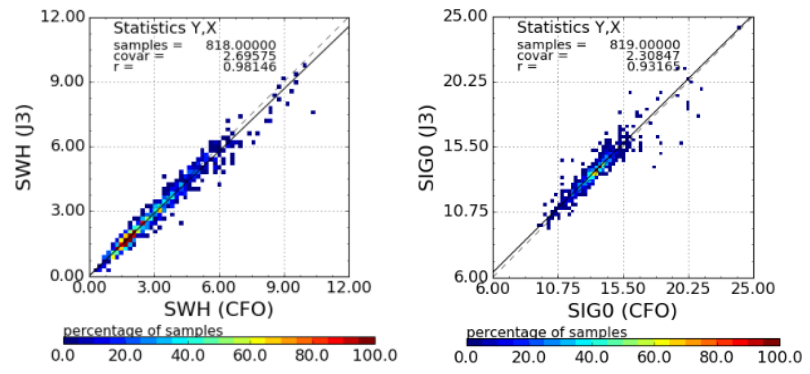

Figure 2: SWIM nadir SWH and $\sigma_{0}$ compared to altimeter Jason 3 at crossover points (time gap $<3 h$ ): left: $S W H$ comparison; right: $\sigma_{0}$ comparison.

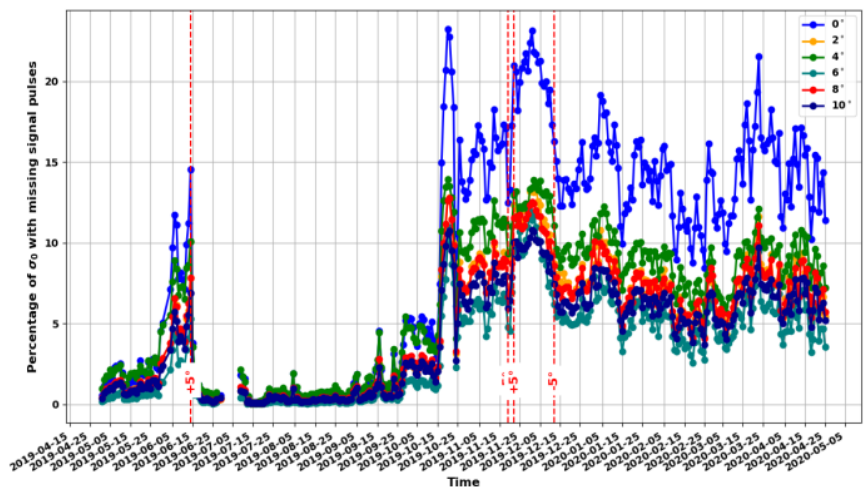

Figure 3: Time series from October 2019 to May 2020 of the occurrence (in \%) of the sigma0 overestimation larger than $1 \mathrm{~dB}$ due to the microcut problem.

\section{NORMALIZED RADAR CROSS-SECTION FOR ALL BEAMS}

The normalized radar cross-section $\sigma_{0}$ is provided for all the SWIM beams from $0^{\circ}$ to $10^{\circ}$ and for all scenes (ocean, seaice, continent). It is provided for each measurement at level $1 \mathrm{~A}$ and averaged at level 2 .

The mean trend of $\sigma_{0}$ obtained from L2 products is shown in Fig.4. Over ocean (Fig.4a) it is globally consistent with GPM data [5]. Over sea-ice (Fig. 4b), it shows a more specular behavior with a rapid decrease from nadir not $10^{\circ}$ incidence.

Fig. 5 illustrates that as expected, the dependency of $\sigma_{0}$ with wind speed at $10^{\circ}$ is low and consistent with the GPM data mean values (less than $1 \mathrm{~dB}$ difference) and trend. It also shows that at light winds (typically less than 4 to $5 \mathrm{~m} / \mathrm{s}$, there are many outliers. To eliminate these case, thresholds will be added in a future version of the processing. In addition, nonhomogenous scenes will be rejected based on a maximum value of $\sigma_{0}$ variances estimated over each footprint after filtering the fluctuation due to waves (scales smaller than $500 \mathrm{~m})$. Fig. 6 shows a map of this filtered $\sigma_{0}$ variance over a 7 -day period for all free ocean scenes. It clearly shows that the highest values of this parameter are preferentially found in tropical regions, in association with either very light winds or rain events.

\section{WAVE SPECTRA}

\subsection{Spectra of the normalized cross-section and speckle evaluation}

The radial spectra of the $\sigma_{0}$ fluctuations are estimated at the Levellb of the processing. During the validation phase, these spectra have been compared to wave spectra from the wave model WW3 (see [3]). The best correlation was obtained for 
SWIM beams at $8^{\circ}$ and $10^{\circ}$ incidences and showed that the range of wavelengths which can be captured cover the one specified for CFOSAT ([70-500 m]). However, an important

(a)

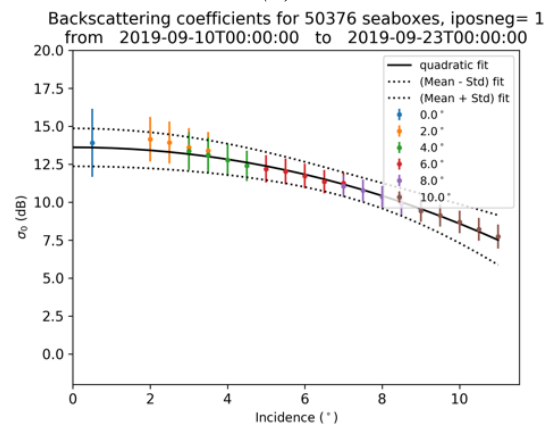

(b)

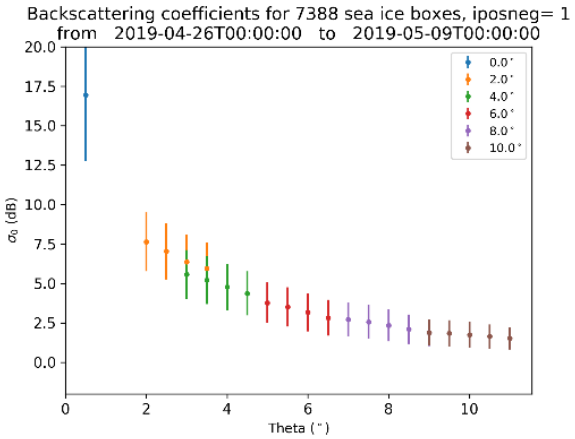

Figure 4. (a) mean profiles of $\sigma o$ with incidence angle from SWIM over free ocean surface (a) and over sea-ice (b).

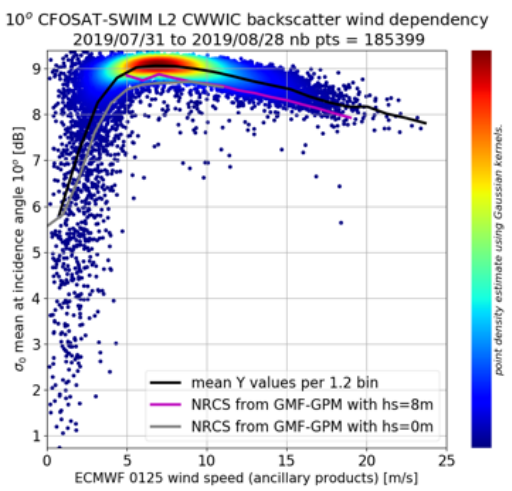

Figure 5: Distribution of $\sigma_{0}$ at $10^{\circ}\left( \pm 0.5^{\circ}\right)$ as a function of ECMWF model wind speed. The colored lines correspond to mean values of GPM data for the same wind (and for two different significant wave heights).

perturbation was evidenced in a $\pm 15^{\circ}$ directional sector around the satellite-track with a maximum of energy of the fluctuations without any relation with the wave spectra. It is attributed to the increase of speckle noise associated to the minimum of Doppler Bandwidth in this direction. In order to correct the fluctuation spectra form this speckle perturbation, an empirical model was built using the SWIM data to parameterize the density spectrum of speckle both within and outside this perturbed angular sector (see [3]). Within the $\pm 15^{\circ}$ angular sector affected by the decrease of radar Doppler bandwidth, the speckle intensity was found to vary with latitude and with sea surface conditions. In opposite, for all other directions, for which the Doppler Bandwidth is larger than the Pulse Repetition Frequency, the speckle noise level is governed by the number of samples averaged in time and in radial distance.

\subsection{Wave spectra and associated parameters}

Directional spectra contain complete spectral information. One way to characterize globally their quality is to analyze the correlation index as proposed by [6] with respect to a reference. This analysis was performed for the wave slope spectra with the MFWAM wave model spectra as reference on a 13-day data set; it led us conclude that the best (resp. lowest) consistency of the directional slope spectra with model spectra are obtained for the $10^{\circ}$ beam (resp. $6^{\circ}$ beam) observations with $68 \%$ of cases with a correlation coefficient larger than 0.5 for this beam (see [3]).

The main parameters of the spectra and of their partitions were also compared with their counterpart provided by MFWAM wave model spectra and from in situ data. This is illustrated with Fig. 7 with scatterplots for the significant wave height, dominant wave direction and dominant wavelength of the $1^{\text {st }}$ spectral partition of SWIM (beam $10^{\circ}$ ) spectra with respect to the $1^{\text {st }}$ swell partition of MFWAM. Overall the agreement is good and shows that SWIM is able to provide mean wave parameters with a good accuracy in most of the cases. Some outliers are however observed for the wave direction and wavelength parameters. They are attributed to remaining impact of the speckle noise maximum in the along-track direction. The overestimation in $\mathrm{SWH}$ for $\mathrm{SWH}<3 \mathrm{~m}$ is probably due to amplification of a remaining noise floor at low wave numbers when converting wave slope spectra to wave height spectra. This is illustrated in Fig 8. A filtering of this noise floor at small wavenumbers will be proposed in the future, while avoiding to filter out swell energy.

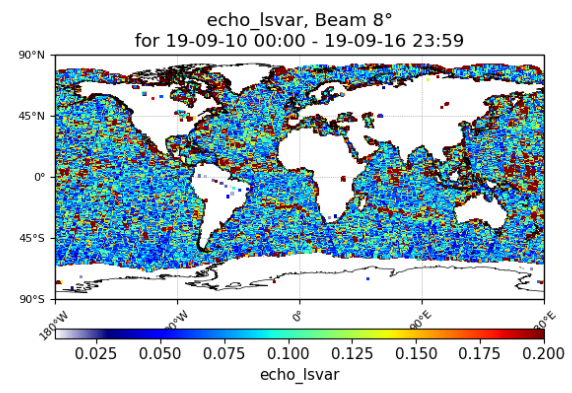

Figure 6: Map of the filtered $\sigma 0$ variance parameter over a 7-day period, for the SWIM data from the $8^{\circ}$ incidence beam. 


\section{CONCLUSIONS}

SWIM is a very innovative instrument which provides both integrated and spectral information on surface ocean waves. The CAL/VAL results prove all its quality even if there are still margins of improvement for the geophysical product retrieval. Despite a lower acquisition rate compared to standard altimeter missions, the nadir beam data $\left(\mathrm{SWH}, \sigma_{0}\right.$ and wind speed) show similar performances with respect to more standard altimeter configurations, thanks to the new "adaptive" retracking algorithm [4]. As for off-nadir data, the trend of $\sigma_{0}$ with incidence and with wind speed is very similar to that provided by GPM data, and the bias with respect to mean GPM values is less or of the order of $1 \mathrm{~dB}$ without any a posteriori calibration. Wave spectra and associated parameters are in good agreement with model data in the wavelength range of interest $(70 \mathrm{~m}$ to $500 \mathrm{~m})$. In spite of a speckle correction parameterized from the SWIM data themselves, a remaining speckle perturbation around the $\pm 15^{\circ}$ sector on each side of the direction of the satellite track slightly degrades the performance for along-track propagating waves. Another impact of the speckle correction uncertainty is an amplification of the noise floor at small wave numbers, which creates spurious peaks in the 1D wave height spectra. These two shortcomings will be the subject of future work to improve the products provided to users.

\section{REFERENCES}

[1] Liu Jianqiang, Wenming Lin, Xiaolong Dong, et al, «First Results From the Rotating Fan Beam Scatterometer Onboard CFOSAT », 10.1109/TGRS.2020.2990708, 2020

[2] Hauser D., C. Tison, T. Amiot, L. Delaye, N. Corcoral et al, SWIM: the first spaceborne wave scatterometer, 10.1109/TGRS.2017.2658672, 2017

[3] Hauser D., C. Tourain, L. Hermozo et al, "New observations from The SWIM radar on board CFOSAT; instrument validation and ocean wave measurement assessment", doi 10.1109/TGRS.2020.2994372, 2020

[4] Tourain C., F. Piras, A. Ollivier et al, "CFOSAT SWIM Adaptive retracking: description and validation", IEEE/TGRS, in revision, 2020

[5] Gressani V., D. Nouguier and A. Mouche, "Wave Spectrometer Tilt Modulation Transfer Function Using Near-Nadir Ku-and KaBand GPM Radar Measurements, Proceedings of the 2018 IEEE IGARSSymposiium, Valencia (Spain), 2018

[6] Hasselmann S., C. Brüning, K. Hasselmann and P. Heimbach, "An improved algorithm for the retrieval of ocean wave spectra from synthetic aperture radar image spectra", Journal of Geophys. Res. Oceans, Vol 101, N07, p16615-16629, 1996
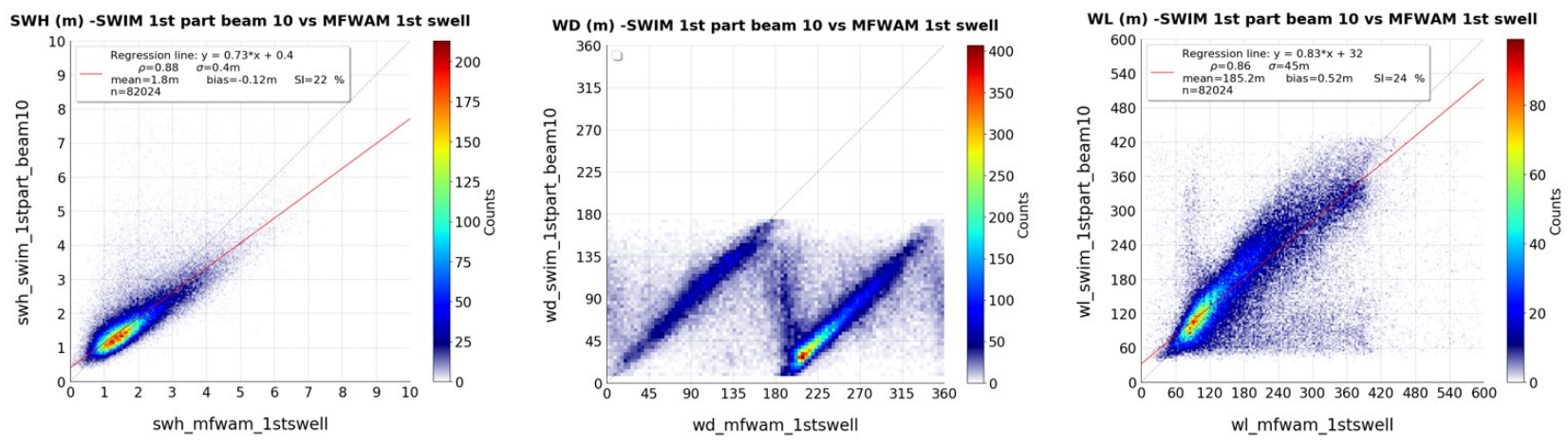

Figure 7: Scatter plots of the first spectral partition parameters from the $10^{\circ}$ beam SWIM data as a function of their counterpart from the MFWAM model, for a 13-day period (26 April-8 May 2019)- (a) Significant wave height; (b) Dominant direction (from North); (c) Dominant wavelength.
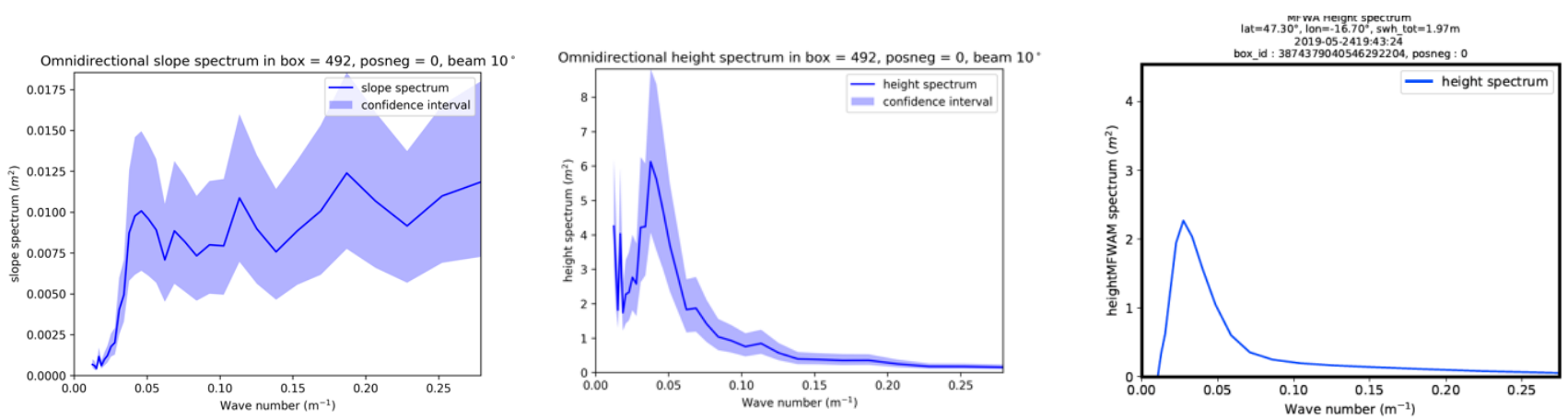

Figure 8: Examples of omni-directional spectrum of wave slope (a) and wave height (b) as a function of wavenumber, with the confidence interval in shaded blue color. The corresponding omni-directional spectrum of wave height from MFWAM is illustrated in (c). 